\title{
A Novel Energy-Efficient Architecture Based on QoS for the Smart Grid
}

\author{
Ali Nabati, Sayyed Majid Mazinani, Member, IACSIT, and Iman Sariri Ajili
}

\begin{abstract}
Today by more complicating in smart grid, request and need for reliability, security and more efficiency and also environmental concerns, we need to change the operation and use of electrical networks. Power companies and the government consider that the smart grid will be salvation for the future. Recent advances in the fabrication of integrated circuits and the development of wireless communication technology that is cusses WSN entry in smart grid. One of the most important features of sensor networks used in smart grid is the energy constraints. So we will offer a new architecture for smart grid in the field of the WSNs to reduce the total energy. Moreover, we will improve some routing factors, QoS parameters such as delay and reliability with respect to the desired user.
\end{abstract}

Index Terms-Smart grid, wireless sensor network, sectoring, minimum spanning tree.

\section{INTRODUCTION}

Recent advances in the fabrication of integrated circuits and the development of wireless communication technology that is cusses WSN entry in smart grid and it based the smart grid according to sensor networks.

Indeed, WSNs in smart grid systems can cause the user to image physically and electrically on WAN, HAN and NAN. A wireless sensor network makes of some sensor nodes that are randomly scattered in an area. This small node have responsibility to sense the sent and received data through current network and then send them to specific destination.after the establishment of the node in the network, they communicate with each other and send the latest information to the base station. Essentially the node constitute by some units such measurement unit, data processing unit, communication unit and power unit .Each of these scattered sensor nodes can collect data and transmit them to other sensors or external stationary sources. A stationary source may be either fixed or animated nodes that connect the sensor network infrastructure, smart grid or Internet connection in order to access the result by users [1].

Recently a lot of research have been carried out in some subject for communication between the sensors data collection and data processing and also management the activities. In most applications, the sensor node, are dependent on energy supply and communication bandwidth. Therefore we need the technologies to eliminate inefficiencies

Manuscript received April 25, 2014; revised July 1, 2014.

Ali Nabati and Sayyed Majid Mazinani are with Imam Reza International University, Mashhad, Iran (e-mail: nabati65@yahoo.com, smajid_mazinani@yahoo.com).

Iman Sariri Ajili is with Iranian Construction Engineering Organization Province of Khorasan Razavi, Iran (e-mail: iman.sariri@yahoo.com). energy that will decrease the network lifetime so increase the efficiency of the limited bandwidth. This limited with the usual number of sensor nodes challenge a lot in design and manage the smart gird that based on WSNs and make the knowledge about energy in all levels is more important than protocols. For example, at the network level, energy cost that can transmit the data from sensors to the station and increase the network lifetime, it's important for us [2]-[4].

On the other hand, a smart grid that based on wireless sensor networks can monitor physical and environmental conditions such as traffic data, the amount of bandwidth consumed, vibration, pressure or motion in various places. To support these applications, smart grid will create a channel for data transmission. This channel has some features such as reliability, fault tolerance and data transfer speeds and in case of failure in crisis qualifications; the channel should continue to maintain these features. This critical qualification may appear because of node failure and loss of direction. Quality of service can be an important way to ensure requirements and also it's necessary for a challenging issue [3]-[5]. Fig. 1 shows the architect of smart grid based on WSN.

\section{PROCEDURE FOR PAPER SUBMISSION}

\section{A. Review Stage}

One of the most important characteristics of wireless sensor networks that used in smart grid is nodes in the network power limitations and often the main priority in this type of networks is the power consumed by nodes. In the proposed method, we use the hierarchical routing protocols because it decrease energy and manage the network scalability too.

\section{B. Final Stage}

So we're going to give a new architecture for smart grid according to WSNs that show this architecture can reduce the energy consumption of the entire networks. In addition we pay attention to some routing parameters, the quality of service such as delay and Reliability [6].

The rest of this paper is organized as follows.

\section{DETAILS OF THE PROPOSED METHOD AND IMPLEMENTATION}

In this section, we will explain each of the steps of the proposed method and also how to implement the goals [7].

\section{A. Select the Cluster Heads}

$\mathrm{LEACH}$ is one of the normal routing Algorithm. LEACH is Energy efficient protocol that consists of clusters before transferring the data to the base station. 


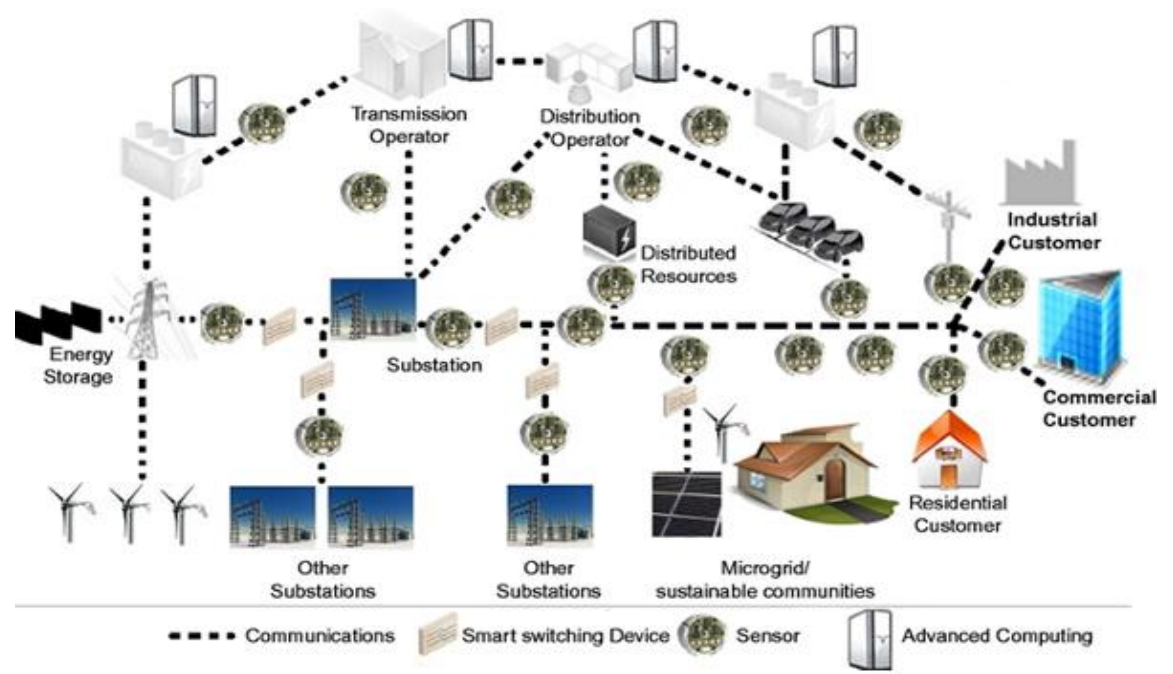

Fig. 1. Architect of smart grid based on WSN.

The working mechanism of this algorithm is that sensor nodes deployed in the sensing area divided into several clusters and each cluster selects a cluster head. After receiving data from all member nodes, the cluster head transmits the data to sink and/or to other cluster heads.

The same sensor nodes with limited energy source work and distribute in dense environments. Sink and base station are far from the network. Given the strength of the signal received from its neighboring nodes it may be possible to distinguish between its own nodes.

\section{1) Basing setting (setp cluster configuration)}

At this stage, cluster heads will determine on a probability function adaptive.at first each node chose 1 or 0 randomly. If the generated number is less than the threshold $T(n)$, the sensor node can be chosen as the cluster head during around. The threshold for determining cluster heads is elected as in (1).

$$
T(\mathrm{n})= \begin{cases}\frac{P}{1-P^{*}\left[\operatorname{rmod}\left(\frac{1}{P}\right)\right]} & \text { if } \mathrm{n} \in \mathrm{G} \\ 0 & \text { otherwise }\end{cases}
$$

$P$ is the desired percentage to become a $\mathrm{CH}, r$ is the current round, and $G$ is the set of nodes that have not being selected as a $\mathrm{CH}$ in the last $1 / P$ rounds.

\section{2) The stable state (normal operation of the network)}

After the $\mathrm{CHs}$ are selected, the $\mathrm{CHs}$ advertise to all sensor nodes in the network that they are the new CHs. Once the sensor nodes receive the advertisement, they determine the cluster to which they want to belong based on the signal strength of the advertisement from the $\mathrm{CHs}$ to the sensor nodes. The sensor nodes inform the appropriate $\mathrm{CHs}$ that they will be a member of the cluster. Fig. 2 shows how to select a cluster head.

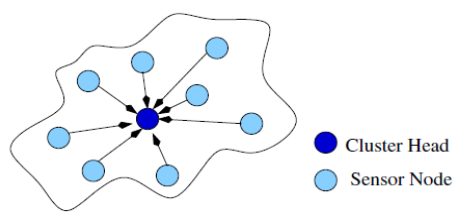

Fig. 2. Cluster representation with star topology [7].

\section{B. Graph Representation}

In smart grid based on wireless sensor networks, except in certain cases the sink sends control information to inform the sensor nodes towards the sink nodes are often used to transfer data. Fig. 3 shows group of cluster head in WSN field. Shortest path (SP) is the small and simple way. The main goal of this algorithm is the subnet graph form so we assume that each node or a router and each edge as a line of communication between the two routers. In this case, each edge a weight basis and it will be possible to calculate the shortest path. SP methods are distance vector protocols, routers best practices in distance to each of the other routers in the network are stored in a vector space. According to the proposed construction of an undirected graph according to how the sensor nodes deployment, data routing operations is required. To construct graph between sensors, we assume the transmission range of a sensor node is ' $d$ '. So the nodes that have geographical distance less then $\mathrm{d}$ will be connected to each other as represented. In Fig. 4 has been shown the graph obtained from WSN field.

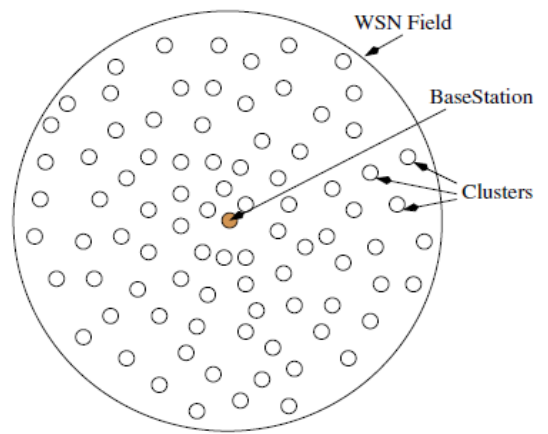

Fig. 3. WSN with the group of cluster [7]

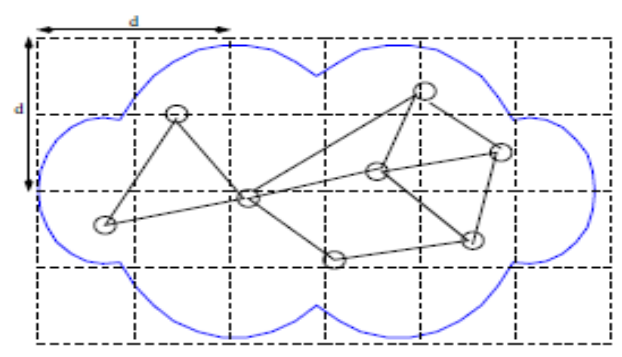

Fig. 4. Graph representation [7]. 


\section{Construction Tree-Based on Efficient Energy Protocol for Sensor Information (TREEPSI)}

If we want to make an algorithm before routing implementation to This flow information (flooding) and also create a loop in the network, leading to inefficient use of resources (especially energy and bandwidth) with a some unnecessary messages to nodes that it will not benefit them. To overcome this problem tree-based protocol for sensor information (TREEPSI) has been proposed. TREEPSI construct a family tree that is a hierarchical routing protocol. With using TREEPSI we can contact to all of created nodes. TREEPSI use the different way to communicate the nodes and make a tree [8]. Fig. 5 shows spanning tree that applied by graph.

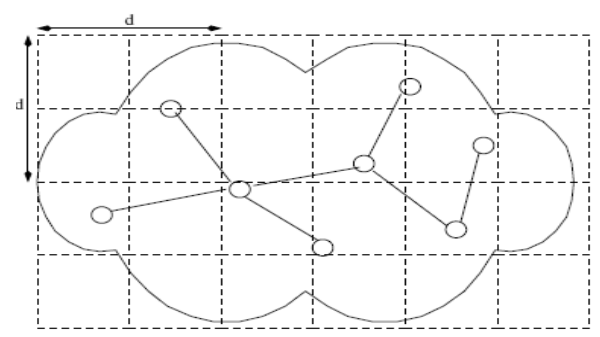

Fig. 5. Spanning tree representation [7].

\section{Sectoring the Area}

According to our research of smart grid based on wireless sensor networks have been done on the levels, and it covered the wide range area so we need the tree that can covered all of the sensors and it will be very large and the management of this tree is really difficult.

The main idea of this part of the research was created to reduce the size of the tree.

By TREEPSI algorithm the total sensor area that have difference of 30 degrees are divided and the base station is origin of all of them.

But for sectoring the area, the base station must have following capabilities:

1) Base station should have transmitters powerful enough so that it can send packets directly to all nodes (single hop).

2) Base station should be able to adjust power of transmission so that nodes at distance greater than specified distance can't receive the transmission.

3) Base station should also have directional antennas which have both capabilities mentioned in points $\mathrm{A}$ and $\mathrm{B}$ above.

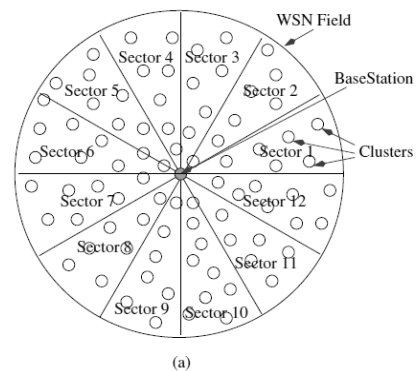

Fig. 6. Sector representation and spanning-tree for a sector [7].

WSN coverage area to other areas that have a $30^{\circ}$ angle it called section and the source of all base stations in each of these areas will be divided. Every unit has a unique SECTOR-ID. SECTOR-ID in order to inform nodes of their information as well as base station, sink package, which contains the information listed is called SBP using directional sends only the nodes are located within the departments are able to get this package. (See Fig. 6).

In this new structure, each cluster heads will lead the packet to only one other neighboring cluster heads so it will avoid the flooding Information and this energy efficiency it realized when we can stop the unnecessary message (Redundant).

\section{E. Leveling the Area}

One of the main advantages of smart grid is implementation improving the services provided to customers.

Another research proposal for improving the quality of service parameters such as the Reliability is delayed by dividing the area into different levels of signal strength and distance from the sink is possible.

Leveling the area has been done by two ways [9]:

1) To divide sensor field into various levels, the base station sends packets containing level ID for level 1 with minimum power level. All the nodes that receive this signal set their levels as 1 . Next the base station increases its signal power to reach the next level and sends packet containing next level ID. All the nodes that receive this signal, if have not already set their level ID, set their level to 2 . This process continues until the base station has sent signals corresponding to all levels. The number of levels into which the network gets divided is equal to the number of different power levels at which the base station has transmitted the signal.

2) Leveling can also be done using hop-count ratio-based technique [9]. In this technique, hop-count of all the nodes is initialized to infinity. First sink broadcasts packets with hop-count field set as zero. Nodes that directly receive these packets set their level as 1 . These updated packets are again broadcast after incrementing hop-count field by 1 . Nodes that receive these packets update their level to 'HopCount +1 ', if their current level is higher than 'HopCount +1 '. The nodes that are having their level equal to or less than the 'HopCount+1' value of the received packet then they don't update their current level value. In wireless communication most of the time Line of sight path is not available, and hence fading problems can occur. In such case hop-count ratio-based technique is preferred.

\section{F. Create Tree for Each Section}

The trees were very large and the network graph by TREEPSI will be very difficult to manage such a large tree. So, after the Portion and Regionalization coverage area for each segment using a spanning tree algorithm to build TREEPSI terms of scale and size of the original tree is smaller.

\section{1) Weight assigned to the edge}

In shorter path method the main objective is to form a graph in which each node is a router and each edge will be a line of communication between the two routers. In this case, each edge a weight basis and it will be possible to calculate the shortest path. Assigning weights in this algorithm, so that the 
two-headed two different levels a and $b$ the amount of weight $\mathrm{a}-\mathrm{b}+1$ condition $(\mathrm{a}>\mathrm{b})$ are at the same level are weighted 1 .

\section{2) Construction of a minimum spanning tree (MST)}

In order to build a spanning tree of the graph produced by the network is a tree which contains all vertices in the graph, but only some of them include the edges [7]. So we can conclude that this description of a network graph over a spanning tree can be produced. minimum spanning tree in graphs where each edge has a weight or value that is being built and it is a tree that if the sum of all graphs that can be obtained by the smallest number in other words, the minimum spanning tree of the graph is a tree network, the trees, spanning the graph, the total weight of its edges, the minimum amount possible. For reach the optimal spanning tree can be used with different algorithms. Three famous algorithms for finding spanning tree are Kruskal's algorithm, Prim's algorithm and Solian's algorithm.

In this research we use Prim's algorithm to reach the MST. This section followed Fig. 7.

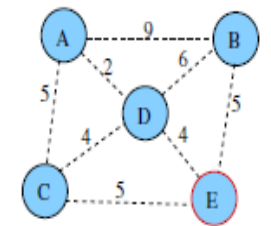

(a)

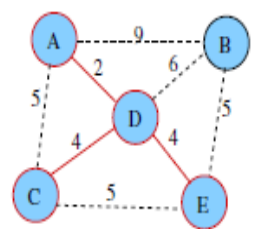

(d)

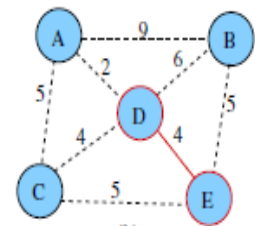

(b)

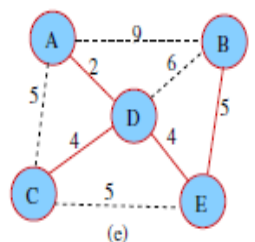

(e)

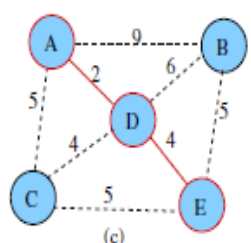

(c)

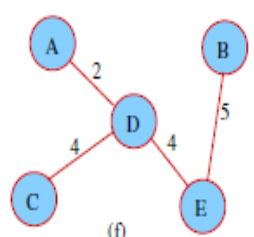

(f)
Fig. 7. Prim's algorithm for MST creation [7].

\section{G. Increased Stability and Fault Tolerance}

One of the most challenging issues in smart grid based on wireless sensor networks and the sustainability debate is fallible Network. The idea of research in this field is thus to increase the stability and fault-tolerance of network nodes on the boundaries of each section there is an MST to the adjacent do so if a node within a sector was in trouble when nodes remaining 2 MST Join your neighbors are amputated is divided into sections.

1) Interconnection between MSTs: the sensor nodes at the boundaries of sectors are also connected with MSTs of neighboring sectors. To identify which nodes belong at boundaries of sector two different approaches are possible:

- During sectoring border nodes would receive SBP of neighboring sectors with reduced signal strength in comparison to received signal strength of their own SBP. Thus nodes which receive SBP with different sector ID scan identify themselves as boundary nodes.

- Base-station can also send separate messages with the help of directional antenna to mark the boundary nodes. Only the nodes which receive this special message can identify themselves as boundary nodes.

For building interconnection between MSTs boundary nodes send message as a request for interconnection,
Receiving node accept this request only if receiving node differs from sending node in both sector ID and level ID. Fig. 8 shows the connection of sector MST to other neighboring sector.

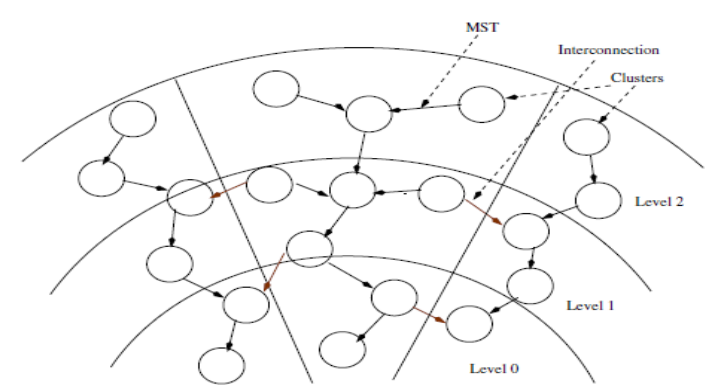

Fig. 8. Connection of sector MST to other neighboring sector [7].

\section{H. Routing / Data Transmission}

Once MST construction is complete, root node of the MST Generates a TDMA schedule for the transmission [10] and circulates it in the MST. Based on the sensed spectrum availability information, base station also assigns the frequency bands available to each of the cluster and sector based MSTs. When nodes are aware of the TDMA schedule and the frequency bands, they transmit data and perform fusion in appropriate time-slots allotted to them. In every slot one or more node transmits gathered data to the lower level nodes. The lower level nodes fuse the received data with sensed data and send the result to the next lower level nodes. The generation of TDMA schedule for MST is described with the help of example. Consider MST generated in Fig. 7(f). Table I shows the edge information of the MST. In Table I, node-ID is ID of transmitting node and Next Hop Node ID is ID of receiving node. So, node $D, E$ participates in the routing and node $A, B, C$ are the leaf nodes. As node ' $A$ ' and ' $C$ ' have the same next hop node, they both can't transmit the data simultaneously [11]. Thus in first round only nodes $A, B$ transmit data and in next round node ' $C$ can transmit its data. In third round node $D$ can transmit fusion result. Thus the sequence of transmissions is $A, B, C$, and then $D$. Fig. 9 shows the time order of the TDMA schedule generated [7].

TABLE I: EDGE INFORMATION OF MST [7]

\begin{tabular}{|c|c|}
\hline Next ID & Next Hop ID \\
\hline $\mathrm{A}$ & $\mathrm{D}$ \\
\hline $\mathrm{B}$ & $\mathrm{E}$ \\
\hline $\mathrm{C}$ & $\mathrm{D}$ \\
\hline $\mathrm{D}$ & $\mathrm{E}$ \\
\hline $\mathrm{E}$ & - \\
\hline
\end{tabular}

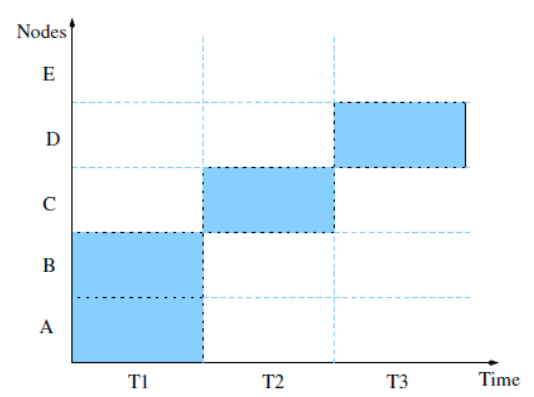

Fig. 9. TDMA schedule order of MST (in mac layer) [7]. 


\section{Improve Service Quality Parameters (QOS)}

Coupling between neighboring sectors together make more fault-tolerant network architecture. Of course, we can consider some of the factors in routing parameters, quality of service such as delay and reliability depending on the intended application improved, so that the trees spanning consists of two section adjacent to the two aspects of various forms one section a general problem in the energy network consists MST as criteria to be considered, while the adjacent section of QoS parameters such as packet delay or reliability as consider optimal spanning tree [12]. The main groups depending on the form of the usual data packet consider requesting QoS .notes through the spanning tree with the appropriate application package, depending on the sink routing and the sector of the package that is produced by a sector of the requested QoS is closed to two levels lower than send out and thus delay to decrease.

\section{ANALYSIS AND RESULTS}

\section{A. Simulation}

Simulations of this paper has been carried out by simulation software opnet Network Simulation of surface dimensions m 200×200 meters an hour is done. And also we used 100 nodes in it.

In this article we compare four scenarios.

\section{B. Analysis and Results}

One of the major challenges for advanced smart grid based on wireless sensor networks is the decreasing energy constraints and lifetime of the network. Also, changes in the routing platform to the base station of the service quality factors, such as delay and reliability have improved, and also we used 100 nodes in it.

\section{First Scenario}

A node (i) put in a random location Considered as an arbitrary node and consider bed manufacturer.

In the first scenario, energy consumption in the tree network built by TREEPSI graph algorithms and then compared to the next scenario, delay and reliability parameters of quality of service parameters have been implemented. This is shown in Fig. 10.

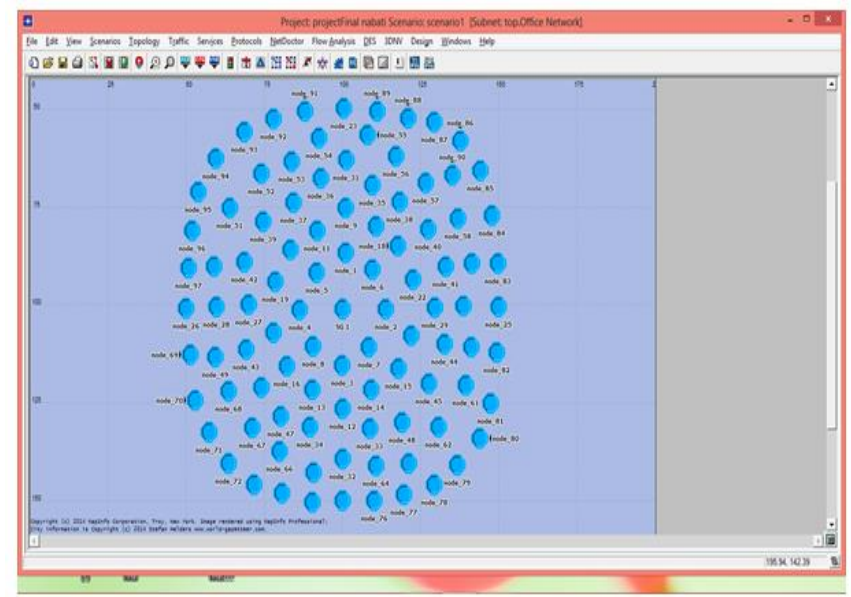

Fig. 10. First scenario.

\section{Second Scenario}

In Fig. 11 sector ideas and graded in second scenario simulated network approach has been applied and we have simulated consumption as well as reliability and delay.

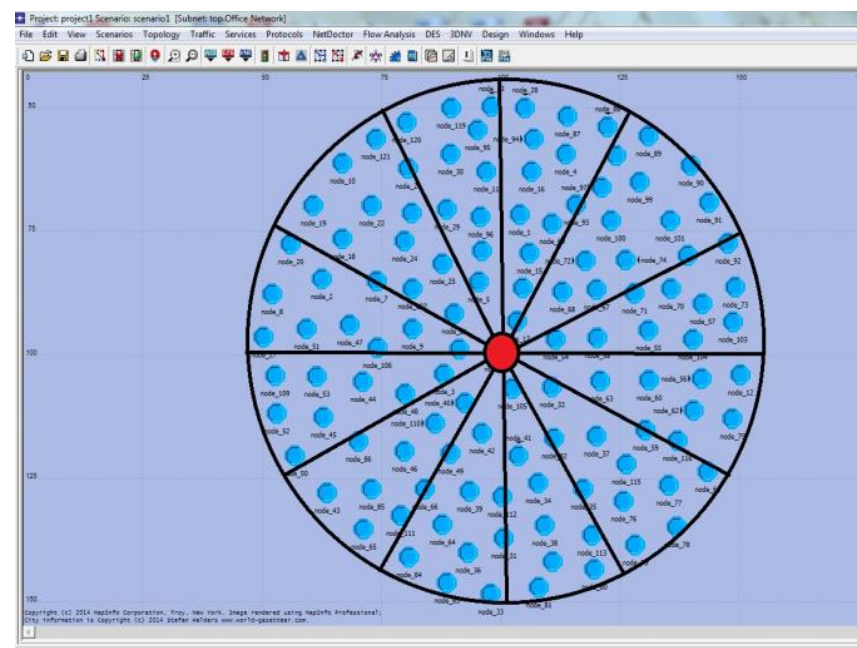

Fig. 11. Second scenario.

\section{E. Third Scenario}

In this scenario we believe the idea that simulate the routing when the light reach to tree and for increase the network stability, it communicate with its light neighbors. But in making this connection are considered priority energy consumption and latency and reliability will be the next priority. So light communicate with its surface light and it will decrease the energy. On the other hand, because the light has to communicate with it from a greater distance is the arrival time and the delay is greater substrate. The entries that described before is shown in Fig. 12.

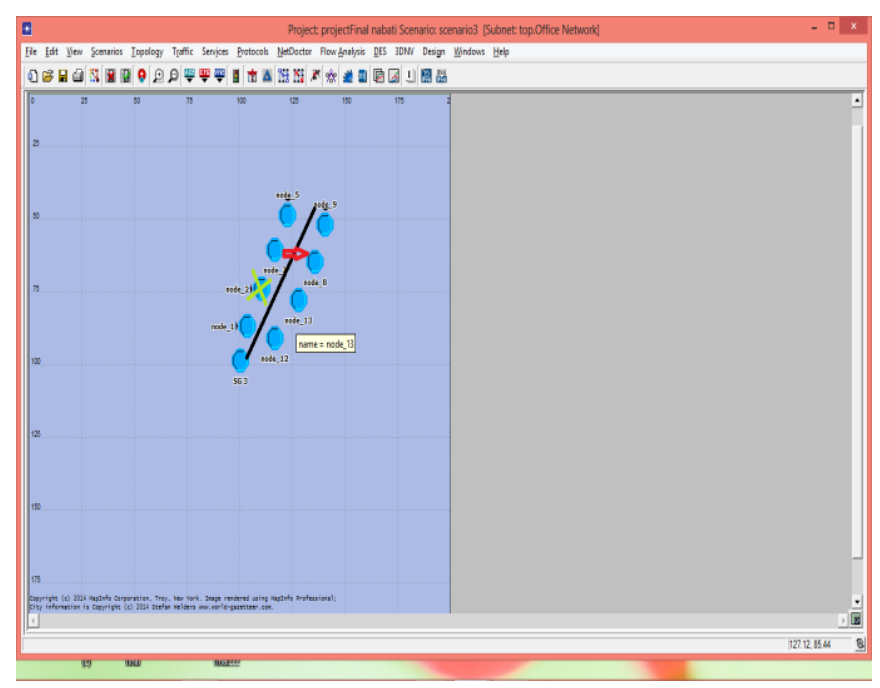

Fig. 12. Third scenario.

\section{F. Scenario IV}

In this scenario, priority routing delay and reliability for the requested Quality of Service and Would quickly be put to the base station and with more energy due to longer fruitful to interact with light that It is located in the lower level and thus further delay will reduce energy cost. Fig. 13 shows how to implement scenario 4 . 


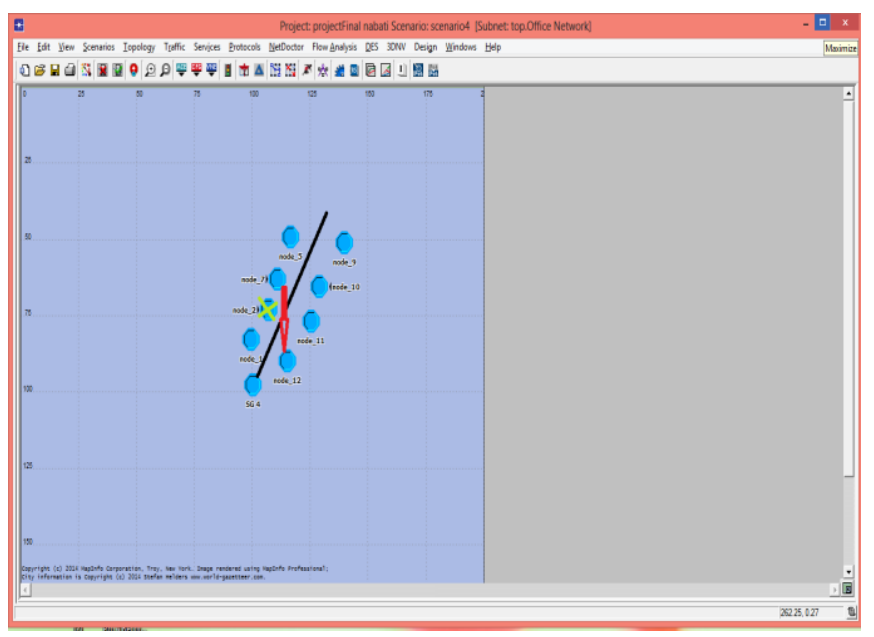

Fig. 13. Scenario IV.

\section{G. Packet Generation Diagram}

We consider scenarios Number and type the same simulation on equal terms. In Fig. 14 shows the packet generation of 4 scenarios.

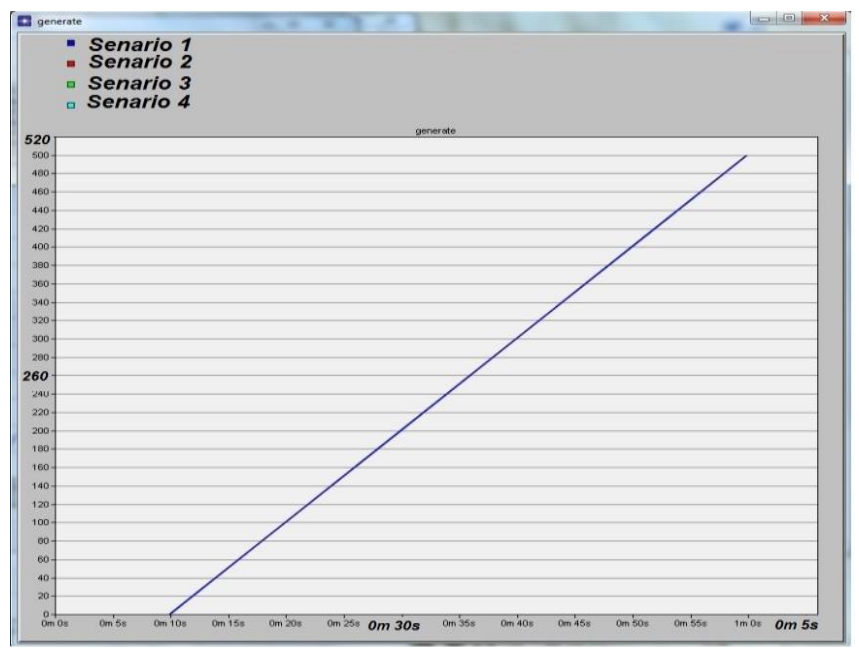

Fig. 14. Packet generation diagram

\section{H. Energy Use Diagram}

Energy diagram is shown in Fig. 15 the second scenario is lowest amount and it because of segmentation. The next level is the third scenario in which energy consumption is a priority and consumes more energy than the light because of a jump is a neighbor. In the third quarter of this figure is the scenario that gives priority to the quality of service parameters.so energy consumption will be higher than in the previous scenario 2 and the first scenario is the most energy the product range covers a large tree in more energy transfer to the bed due to the longer path.

\section{Delay Diagram}

The diagram of the base station is delayed hospital arrival. Because of the large tree naturally produced in the first scenario longer going to consider the context of the destination node, the base station is reached. The second scenario because of a covering of the door caused the rising transition delay. The idea of segmenting the second scenario also has a large impact on the delay and the delay is less than the first scenario. The fourth scenario also prioritize delay of three scenarios with more speed before reaching the destination platform to jump up and more energy will be.
Considering the delay curves we find that the first packet to the base station is received later than the first scenario. Thus began the first scenario, all scenarios Chart delayed starts. The entries that explained before is shown in Fig. 16.

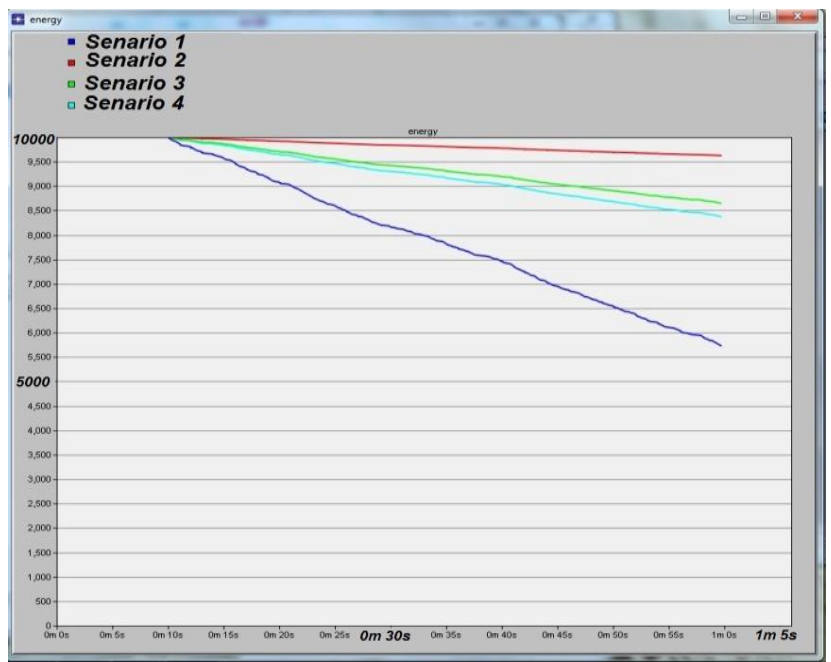

Fig. 15. Energy consumption diagram.

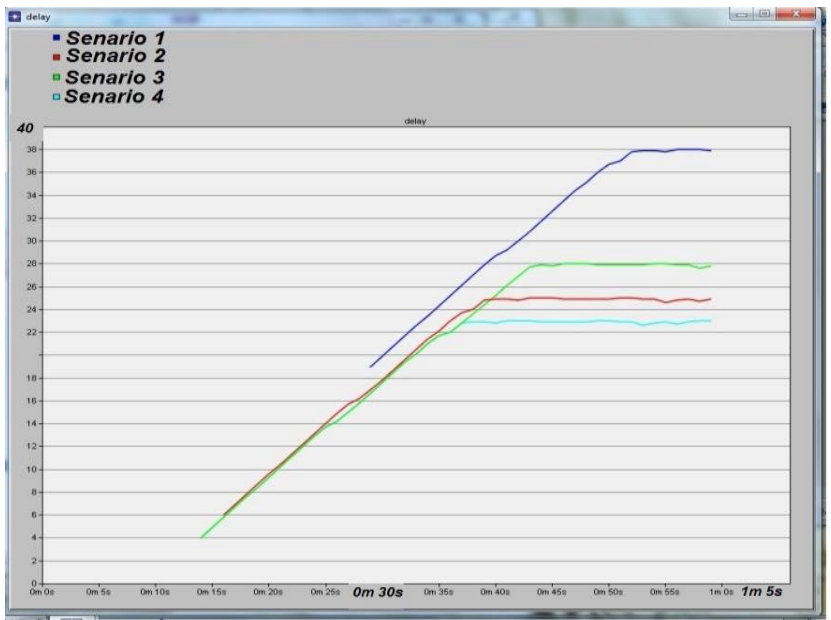

Fig. 16. Delay diagram.

\section{J. Jitter Diagram}

Delay oscillation in the delay between every moment with their before moment that exactly according to diagram delay oscillation diagrams is justified. Fig. 17 related to jitter diagram.

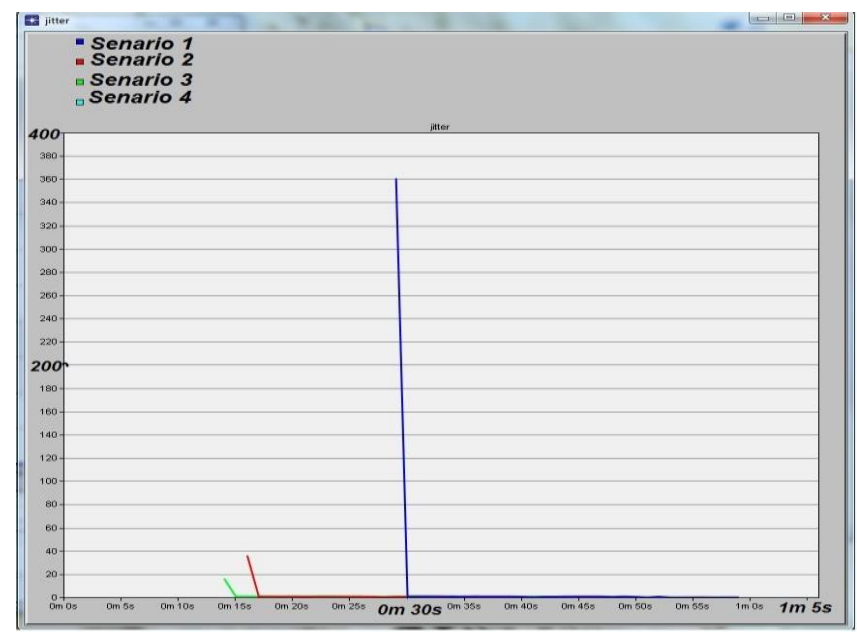

Fig. 17. Jitter diagram 


\section{K. Reliability Diagram}

As you can see in Fig. 18 whatever was jumping on a bed under the direction of the path jump higher reliability is less passes. The fourth scenario is the explanation given to the number of beds Number of beds produced more reliable. The second and third scenarios reliability is pretty close together. In the first scenario, both because of reliability and a long path routing tree is much less three-dimensional scenario.

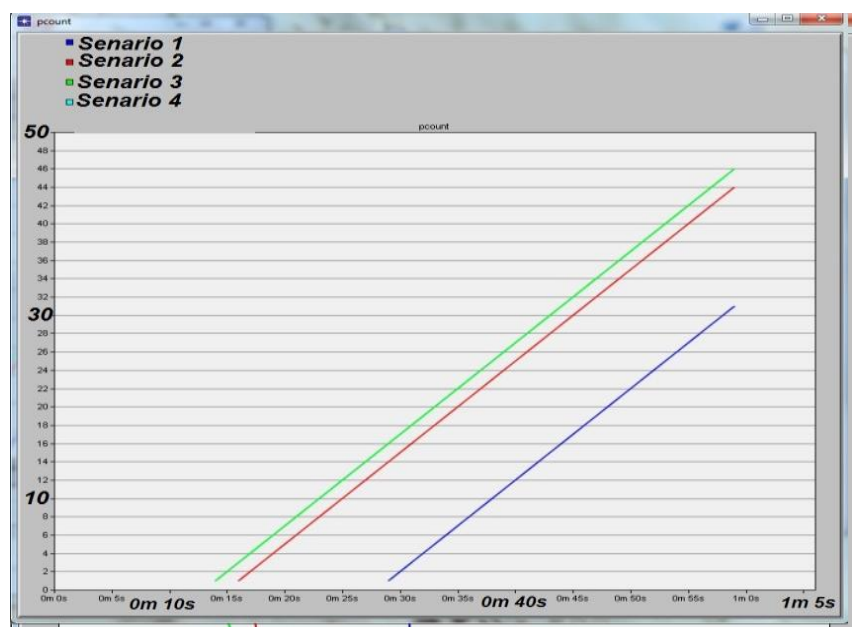

Fig. 18. Reliability diagram.

\section{CONCLUSION}

In this paper we have given a new architecture for data transfer with the energy efficiency of the network with minimum spanning tree (MST). At the beginning of this paper we described the smart grid in wireless sensor networks and we have shown the multi-level fault tolerance and also we have discussed the Simple set of data transfer methods. This architecture makes data collection and data transmission to minimize and decrease the total energy that used in network with stop flooding Information delete unnecessary messages. Regarding the very important issue of energy limitation and avoid wasting it, we decided to examine the routing algorithms from different directions, especially energy consumption.

\section{REFERENCES}

[1] S. Lohani, "Communication network analysis in smart grid," Student Thesis, School of Computer Sciene, Phisics and Mathematics, pp. 1-63, 2012.

[2] J. Yick, "Wireless sensor network survey,"in Proc. IEEE Conf. on Computer Networks, 2008, pp. 2292-2330.

[3] Y. Wangand, W. Lin, and T. Zhang, "Study on security of wireless sensor networks in smart grid," in Proc. International Conference on Power System Technology, 2010, pp. 1-7.

[4] I. F. Akyildiz, "A survey on wireless multimedia sensor networks," in Proc. Conference on Computer Networks, 2007, pp. 921-960.

[5] E. Kantarci and M. Mouftah, "Wireless sensor networks for smart grid applications," in Proc. International Conference on Electronics, Communications and Photonics, 2011, pp. 1-6.
[6] V. C. Gungor, "Opportunities and challenges of wireless sensor networks in smart grid - A case study of link quality assessments in power distribution systems," IEEE Transactions on Industrial Electronics, pp. 1-8, 2010.

[7] D. Singhal, S. Barjatiya, and G. Ramamurthy "A novel network architecture for cognitive wireless sensor network," in Proc. International Conference on Signal Processing, Communication, Computing and Networking Technologies, 2011.

[8] S. S. Satapathy and N. Sarma, "TREEPSI: Tree based energy efficient protocol for sensor information in wireless and optical communications networks," in Proc. 2006 IFIP International Conference, 2006.

[9] A. Mirza and R. M. Garimella, "PASCAL: Power aware sectoring based clustering algorithm for wireless sensor networks," in Proc. International Conference of Information Networking (ICOIN 2009), 2009, pp. 1-6.

[10] N. Saputro, K. Akkaya, and S. Uludag, "A survey of routing protocols for smart grid communications," Elsevier Journal on Computer Networks, vol. 56, no. 11, pp. 2742-2771, 2012.

[11] B. E. Bilgin and V. C. Gungor, "On the performance of multi-channel wireless sensor networks in smart grid environments," in Proc. 20th International Conference on Computer Communications and Networks, 2011, pp. 1-6.

[12] O. Younis and S. Fahmy, "Heed: A hybrid, energy-efficient, distributed clustering approach for ad hoc sensor networks," Mobile Computing IEEE Transactions, vol. 3, no. 4, pp. 366 - 379, Oct.-Dec. 2004.

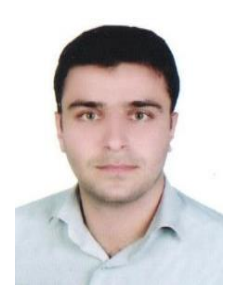

Ali Nabati was born in Gorgan, Iran on September 5, 1986. He received the B.S. degree in Information Communication Technology (ICT) Engineering from ITMC University of Shiraz, Iran in 2010. He is a M.A. student in telecommunication engineering in Imam Reza International University, Mashhad, Iran. He is also the head of engineer's data center in Telecommunication Infrastructure Company in Gorgan, Iran. His research interests include computer networks, wireless sensor networks and smart grids.

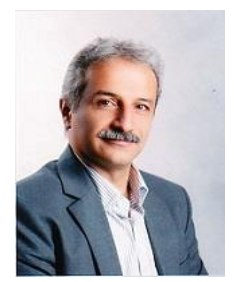

Sayyed Majid Mazinani was born in Mashhad, Iran on January 28, 1971. He received his bachelor degree in electronics from Ferdowsi University, Mashhad, Iran in 1994 and his master degree in remote sensing and image processing from Tarbiat Modarres University, Tehran, Iran in 1997. He worked in IRIB from 1999 to 2004. He also received his $\mathrm{PhD}$ in wireless sensor networks from Ferdowsi University, Mashhad, Iran in 2009. He is currently an assistant professor at the Faculty of Engineering in Imam Reza International University, Mashhad, Iran. He was the head of Department of Electrical and Computer Engineering from 2009 to 2012. His research interests include computer networks, wireless sensor networks and smart grids.

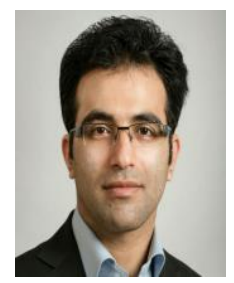

Iman Sariri Ajili received the B.S. degree in electrical engineering from Azad University, Gonabad, Iran, in 2004 and the M.S. degree in control engineering from Azad University, Gonabad, Iran, in 2008. From 2008 to 2009, he was a research assistant with the Institute of Data Mining, Sanaye Behshahr, Tehran, Iran. His research interest includes the development of data mining in smart grids, decision management system. making with advanced data-mining in building 